\title{
Linx
}

Revue des linguistes de l'université Paris X Nanterre

$58 \mid 2008$

Aspects de comme

\section{Analogie et coordination en comme}

\section{François Mouret et Marianne Desmets}

\section{OpenEdition}

\section{Journals}

Édition électronique

URL : http://journals.openedition.org/linx/329

DOI : $10.4000 /$ linx.329

ISSN : 2118-9692

\section{Éditeur}

Presses universitaires de Paris Nanterre

\section{Édition imprimée}

Date de publication : 1 juin 2008

Pagination : 75-96

ISSN : 0246-8743

\section{Référence électronique}

François Mouret et Marianne Desmets, « Analogie et coordination en comme », Linx [En ligne],

58 | 2008, mis en ligne le 16 février 2011, consulté le 19 avril 2019. URL : http://

journals.openedition.org/linx/329 ; DOI : 10.4000/linx.329 


\title{
Analogie et coordination en comme
}

\author{
François Mouret \\ Université Rennes 2- UMR 7110- LLF \\ E Marianne Desmets \\ Université Paris Ouest-Nanterre- \\ UMR 7110- LLF E UMR 7114-Modyco
}

\section{Introduction}

C'est un fait bien repéré dans les grammaires que le mot comme peut, lorsqu'il relie deux $\mathrm{SN}$ sujets, donner lieu à deux types d'accord (voir par exemple Grévisse \& Goosse 2008, \$454). Le verbe s'accorde avec le premier sujet réalisé lorsque la séquence introduite par comme est prosodiquement «incidente » au sens de Bonami $e t$ al. (2004), c'est-à-dire non intégrée à la mélodie de la phrase (1a), tandis qu'il s'accorde, semble-t-il, avec la construction $S N$ comme $S N$ dans son ensemble lorsque la séquence est au contraire prosodiquement intégrée (1b), à l'image d'une coordination additive $(1 \mathrm{c})^{2}$.

(1) a. La France, comme l'Angleterre, est favorable à ce projet.

\footnotetext{
${ }^{1}$ Nous remercions Anne Abeillé pour ses commentaires, ainsi qu'un relecteur anonyme.

${ }^{2}$ Nous ne chercherons pas à préciser davantage ici les propriétés phonético-phonologiques de l'incidence. Voir notamment Delais-Roussarie (2005, 2008). Nous régularisons dans cet article l'usage écrit en encadrant systématiquement par des virgules le constituant incident.
} 
François Mouret \& Marianne Desmets

b. La France comme l'Angleterre sont favorables à ce projet.

c. La France et l'Angleterre sont favorables à ce projet.

Les tours incidents du type (1a) ont été bien étudiés : il s'agit de constructions comparatives elliptiques ajouts à la phrase (sans conséquence de fait sur l'accord du verbe avec le sujet) appelées « comparatives d'analogie », parce qu'elles établissent une relation d'analogie, ou encore de similarité, entre deux propositions (cf. Le Goffic 1991, Desmets 2001, Moline 2008). Les tours intégrés du type (1b), en revanche, soulèvent plus de questions : l'accord au pluriel, qui est bien attesté (cf. (2)), constituet-il un simple effet d'intégration sémantique dû au parallélisme des sujets des deux propositions de la comparaison ou bien faut-il admettre l'existence d'une structure coordonnée et réanalyser, dans ce cas, le mot comme, le tour dans lequel il entre et la contribution sémantico-pragmatique de l'un et de l'autre ?3

(2) a. Chez Renault, la direction comme les syndicalistes reconnaissent que, l'automne dernier, le conflit de Cléon et du Mans avait révélé l'urgente nécessité de donner «de la chair » à un «accord à vivre », certes novateur, mais ressenti comme trop abstrait. (LM)

b. Un professionnel «incontestable » serait nommé à la tête de la Cinq et la société des journalistes comme l'association des téléspectateurs y seraient associées. (LM)

c. Le Maroc comme la Tunisie entrent en effet dans la phase finale de leurs plans d'ajustement qui doit conduire à une libéralisation plus complète de leurs échanges et à la convertibilité de leurs monnaies. (LM)

d. Il reste un bon moyen de s'attacher de la main-d'oeuvre quand la pénurie sévit, et le bâtiment comme les travaux publics ont pour cette raison renoncé à leurs projets de réforme. (LM)

Nous voudrions argumenter dans cet article en faveur d'une analyse intermédiaire. Sur le plan syntaxique, les tours intégrés doivent bel et bien être considérés comme des structures coordonnées avec une recatégorisation de l'adverbe comme en conjonction ( $\$ 1-2)$. Sur le plan interprétatif, en revanche, la construction coordonnée conserve certains traits sémantiques de l'ajout comparatif. La différence se situe au niveau pragmatique : alors que la contribution comparative relève du commentaire en arrière plan, la contribution de la coordination appartient au contenu principal asserté (\$3). De façon intéressante, ce double fonctionnement ne s'étend pas tel quel aux autres formes similatives (ainsi que, de même que). Nous en faisons brièvement la démonstration (\$4) avant de conclure.

\footnotetext{
${ }^{3}$ Les exemples suivis de la mention LM proviennent du journal Le Monde. Ils ont été extraits du Corpus Arboré de Paris 7 (Abeillé et al. 2003). Nous remercions à cet effet Clément Plancq pour son assistance technique.
} 


\section{Propriétés lexicales}

Considérons d'abord la catégorie du mot comme. Nous admettons sans discuter ici qu'il s'agit d'un adverbe qu-dans les constructions comparatives, dont relèvent les tours incidents du type (1a) (voir Desmets 2001). Outre le contraste d'accord, plusieurs propriétés confirment bien qu'il s'agit en revanche d'une conjonction de coordination dans les tours intégrés du type (1b). Nous en retiendrons trois ici, qui concernent la combinabilité des conjonctions entre elles, la position de certains adverbes et l'ordre des mots.

\subsection{Combinabilité avec les conjonctions}

Il est bien connu que les conjonctions telles que $e t$, ou, ni ne sont pas combinables entre elles, sauf cas de lexicalisation (et/ou) (cf. Bègue 1977). C'est ce qui explique par exemple qu'à côté de (3a), qui met en jeu une coordination disjonctive enchâssée dans une coordination conjonctive, on n’a pas (3b). On vérifie en (4) que le mot comme se comporte de façon analogue dans les tours intégrés : il ne se combine pas avec une autre conjonction.

(3) a. Nul doute que la France d'une part et l'Angleterre ou l'Espagne d'autre part sont favorables à ce projet.

b. *Nul doute que la France d'une part et l'Angleterre ou et l'Espagne d'autre part sont favorables à ce projet.

(4) a. Nul doute que la France comme l'Angleterre et l'Espagne sont favorables à ce projet.

b. *Nul doute que la France comme l'Angleterre et comme l'Espagne sont favorables à ce projet.

La situation est différente dans les tours incidents, où comme se comporte exactement comme les autres adverbes que l'on rencontre à l'initiale de la phrase : il peut être précédé d'une conjonction (5b-6b) et ne peut (à la différence des complémenteurs, voir Desmets, 2001 : 54) être remplacé par que (5c-6c) ${ }^{4}$ :

(5) a. Nul doute que la France, comme l'Angleterre et l'Espagne, est favorable à ce projet.

b. Nul doute que la France, comme l'Angleterre et comme l'Espagne, est favorable à ce projet.

c. *Nul doute que la France, comme l'Angleterre et que l'Espagne, est favorable à ce projet.

\footnotetext{
${ }^{4}$ On utilise ici une forme syntaxiquement proche des ajouts comparatifs en comme: il s'agit d'une subordonnée causale ajout à la phrase matrice ; c'est aussi une phrase à extraction qui comporte un adverbe de degré extrait (cf. Desmets, à paraître).
} 
François Mouret \& Marianne Desmets

(6) a. Il ne peut plus bouger, tellement / tant il a peur et il est fatigué.

b. Il ne peut plus bouger, tellement / tant il a peur et tellement / tant il est fatigué.

c. *Il ne peut plus bouger, tellement / tant il a peur et qu'il est fatigué.

\subsection{Position de certains adverbes}

Une autre propriété typique des conjonctions, notée notamment par Piot (1993), est qu'elles peuvent être suivies mais jamais précédées par certains adverbes focalisants (aussi, seulement, ...), mais aussi énonciatifs (en effet, d'ailleurs, ...) ou encore connecteurs (ensuite, alors, ...) :

(7) a. La France et en effet l'Angleterre sont favorables à ce projet.

b. *La France en effet et l'Angleterre sont favorables à ce projet.

Aucune restriction de ce type ne pèse en revanche sur les adverbes, comme l'illustrent les exemples suivants :

(8) a. Il ne peut plus bouger, tellement / tant en effet il a peur.

b. Il ne peut plus bouger, en effet tellement / tant il a peur.

À nouveau, on observe un fonctionnement différentiel du mot comme suivant que le syntagme qu'il introduit est prosodiquement intégré ou non. Il se comporte comme les conjonctions dans le premier cas (9) tandis qu'il se comporte comme un les adverbes dans le second cas $(10)^{5}$.

(9) a. La France comme en effet l'Angleterre sont favorables à ce projet.

b. *La France en effet comme l'Angleterre sont favorables à ce projet.

(10) a. La France, comme en effet l'Angleterre, est favorable à ce projet.

b. La France, en effet comme l'Angleterre, est favorable à ce projet.

\subsection{Contraintes d'ordre}

Considérons enfin certaines contraintes d'ordre. On sait que les syntagmes introduits par une conjonction ne peuvent jamais être antéposés à l'initiale de la phrase $(\text { cf. }(11))^{6}$, à la différence des syntagmes introduits par un adverbe qui le peuvent

${ }^{5}$ Concernant les adverbes, on ajoutera un contraste supplémentaire qui distingue nettement les deux tours. La comparative incidente, qui s'analyse comme un syntagme adverbial (Desmets 2001), peut être modifiée par l'adverbe tout, ce qui n'est pas de cas du conjoint en comme (cf. ia vs ib) :

(i) a. La France, tout comme l'Angleterre, est favorable à ce projet.

b. *La France tout comme l'Angleterre sont favorables à ce projet.

${ }^{6}$ Nous considérons qu'il s'agit là d'une propriété attachée aux conjonctions et non à la structure coordonnée dans son ensemble. En effet, on observe le même blocage lorsque le syntagme introduit 
parfois. Par exemple, les formes introduites par tant ou tellement considérées supra sont assez mauvaises dans cette position $(12 \mathrm{a}, \mathrm{b})$, mais non les comparatives scalaires incidentes étudiées par Amsili et al. (à paraître) (cf. (12c,d)).

(11) a. La France et l'Angleterre sont favorables à ce projet.

b. *Et l'Angleterre, la France sont favorables à ce projet.

(12) a. Il ne peut plus bouger, tellement / tant en effet il a peur.

b. ??'Tellement / tant il a peur, il ne peut plus bouger.

c. Il faut donner du travail aux jeunes, plus que des subventions aux cités.

d. Plus que des subventions aux cités, il faut donner du travail aux jeunes.

À nouveau, le mot comme se comporte comme une conjonction dans les tours intégrés, mais non dans les tours incidents ${ }^{7}$ :

(13) a. La France comme l'Angleterre sont favorables à ce projet.

b. *Comme l'Angleterre, la France sont favorables à ce projet.

(14) a. La France, comme l'Angleterre, est favorable à ce projet.

b. Comme l'Angleterre, la France est favorable à ce projet.

Nous concluons qu'il faut effectivement distinguer deux entrées lexicales suivant que la prosodie est intégrée ou incidente : un comme conjonctif et un comme adverbe introducteur de comparative.

\section{Propriétés de structure}

Nous avons établi la nécessité de recatégoriser l'adverbe comme en conjonction additive dans les tours intégrés. Partant, il est naturel de penser qu'on a affaire dans ce cas à une construction coordonnée distincte des tours incidents qui fonctionnent comme des ajouts à la phrase. Nous présentons deux arguments syntaxiques confirmant cette analyse avant d'examiner plus en détail les structures internes en jeu.

\subsection{Distribution des $S N$ sans déterminant}

Considérons d'abord la distribution des SN sans déterminant. On sait que ces derniers sont exclus de manière générale en fonction sujet ou objet à moins d'être coordonnés (cf. Blanche-Benveniste \& Chervel 1966, Curat 1999, Roodenburg 2005) ${ }^{8}$ :

par la conjonction fonctionne comme un ajout incident à la phrase (cf. Abeillé 2005 et ici même, section $\$ 4)$ :

(i) Paul a, et tout le monde le sait, échoué à l'examen.

(ii) *Et tout le monde le sait, Paul a échoué à l'examen.

${ }^{7}$ La mobilité de la comparative d'analogie dans la phrase matrice (cf. 14) est typique de sa fonction d'ajout à la phrase. 
(15) a. [Médecins et infirmiers] sont prêts à venir travailler le dimanche.

b. Il faut disposer d'un compte bancaire pour toucher [salaire ou retraite].

c. *[Médecins] sont prêts à venir travailler le dimanche.

d. *Il faut disposer d'un compte bancaire pour toucher [salaire].

Il s'agit là d'une propriété attachée à la construction coordonnée. La présence d'une conjonction ne constitue en effet ni une condition nécessaire pour légitimer cet emploi particulier du SN, puisqu'il est autorisé dans les juxtapositions (cf. (16a) ni une condition suffisante, puisqu'il est exclu lorsque le syntagme introduit par la conjonction fonctionne comme un ajout incident au sens d'Abeillé (2005) (cf. (16b,c)).

(16) a. Médecins, infirmiers, aide-soignants sont prêts à venir travailler le dimanche.

b. Les médecins sont prêts, et les infirmiers aussi, à venir travailler le dimanche.

c. *Médecins sont prêts, et infirmiers aussi, à venir travailler le dimanche.

Or, les SN sans déterminant sont autorisés dans nos tours intégrés en comme, tandis qu'ils sont exclus dans les tours incidents correspondants :

(17) a. Médecins comme infirmiers sont prêts à venir travailler le dimanche.

b. *Médecins, comme d'ailleurs infirmiers, sont prêts à venir travailler le dimanche.

Ce contraste s'explique naturellement si la structure s'analyse comme une construction coordonnée dans le premier cas mais non dans le second.

\subsection{Phénomènes d'extraction}

Considérons à présent les phénomènes d'extraction. Il existe une contrainte caractéristique des constructions coordonnées (cf. Ross 1967) selon laquelle on ne peut extraire hors d'un conjoint sans extraire simultanément un constituant parallèle hors des autres, ainsi qu'on peut l'observer en (18) où le symbole - note le complément du nom manquant ${ }^{9}$.

\footnotetext{
${ }^{8}$ Nous simplifions ici quelque peu la distribution. Outre leur emploi dans les coordinations, les SN sans déterminant sont possibles en fonction argumentale dans les citations (i), les constructions à verbe support (ii), les contructions $\mathrm{N}$ prep $\mathrm{N}$ (iii), ainsi qu'avec un mot comparatif dans les contextes à polarité négative (iv).

(i) «Soleil » est un mot masculin en français.

(i) Décision a été prise d'abandonner ce projet.

(iiii) Il a bu bière sur bière.

(iv) Vous ne trouverez pas hôtel plus chic dans la région.

${ }^{9}$ On sait que cette contrainte peut être violée en anglais lorsque la coordination met en jeu une relation de discours causale avec un effet contraire aux attentes (i), une relation de conséquence (ii) ou une relation de succession (iii) (cf. Ross 1967, Goldsmith 1986, Lakoff 1986 et plus récemment Kehler 2002).
} 
(18) a. Reste donc une entreprise dont [le président - et [le vice-président -] se plaisent à vanter le chiffre d'affaire.

b. *Reste donc une entreprise dont [le président -] et [son vice-président] se plaisent à vanter le chiffre d'affaire.

c. *Reste donc une entreprise dont [son président] et [le vice-président -] se plaisent à vanter le chiffre d'affaire.

Crucialement, cette contrainte n'est pas observée par les constructions qui combinent une tête et un ajout, et en particulier dans les comparatives (voir Desmets, ce numéro), puisqu'il est possible d'extraire dans ce cas un constituant de la phrase matrice sans extraire corrélativement un constituant correspondant dans l'ajout (19a). L'extraction hors de l'ajout, par contraste, est exclue (19b), à moins qu'elle n'opère également hors de la principale $(19 \mathrm{c})^{10}$.

(19) a. Reste donc une entreprise dont tous les dirigeants -, [excepté son président], se plaisent à vanter le chiffre d'affaire.

b. *Reste donc une entreprise dont tous ses dirigeants, [excepté le président -], se plaisent à vanter le chiffre d'affaire.

c. Reste donc une entreprise dont tous les dirigeants -, [excepté le président -], se plaisent à vanter le chiffre d'affaire.

On vérifie que nos tours se comportent comme des coordinations lorsque la séquence introduite par comme est prosodiquement intégrée tandis qu'ils se comportent comme des structures tête-ajout lorsque cette même séquence est prosodiquement incidente. Seule l'extraction parallèle est autorisée dans le premier cas (20), contrairement au second cas où l'on peut extraire un constituant hors (du sujet) de la principale et de l'ajout (21a), ou bien uniquement hors (du sujet) de la principale (21b), mais non uniquement hors de l'ajout (21c).

(i) How much can you [drink _ ] and [not end up with a hangover the next morning] ? (Goldsmith $1986: 135)$

(ii) That's the stuff that the guys in the Caucasus [drink _] and [live to be a hundred]. (Lakoff 1986 : 156)

(iii) Here's the whiskey I [went to the store] and [bought_ ]. (Ross 1967 : 94)

La situation est différente en français, comme l'illustre l'acceptabilité dégradée des exemples suivants (cf. Mouret 2007) :

(iv) ??Je me demande combien de bières on peut boire et quand même rester sobre.

(v) ${ }^{*}$ C'est le genre de régime que Marie aimerait bien [faire _ ] et [enfin rentrer dans sa robe].

(vi) *Voici le whisky que Paul [est allé au supermarché] et [a acheté _ ].

${ }^{10}$ Cette dernière possibilité, connue sous le nom d'extraction parasite, est contrainte en français. Elle est soumise à variation dans les infinitives (i) et interdite (pour tous les locuteurs) lorsque l'ajout est non fini (ii).

(i) \%C’est un livre qu'il a soigneusement rangé - après avoir lu -.

(ii) *C'est un livre qu'il a soignement rangé - après qu'il a lu -. 
(20) a. Reste donc une entreprise dont [le président -] comme [le vice-président -] se plaisent à vanter le chiffre d'affaire.

b. *Reste donc une entreprise dont [le président -] comme [son vice-président] se plaisent se à vanter le chiffre d'affaire.

c. *Reste donc une entreprise dont [son président] comme [le vice-président -] se plaisent à vanter le chiffre d'affaire.

(21) a. Reste donc une entreprise dont le président -, [comme d'ailleurs le viceprésident -], se plait à vanter le chiffre d'affaire.

b. Reste donc une entreprise dont le président -, [comme d'ailleurs son viceprésident], se plait se à vanter le chiffre d'affaire.

c. *Reste donc une entreprise dont son président, [comme d'ailleurs le viceprésident -], se plait à vanter le chiffre d'affaire.

Nous concluons qu'il faut bel et bien distinguer deux constructions syntaxiques: l'une est analysable comme une coordination, l'autre comme une structure tête-ajout.

\subsection{Structures internes}

Ayant montré que nous avons affaire à deux constructions distinctes, le détail de leur structure interne constitue un problème relativement orthogonal, dans la mesure où l'analyse des structures coordonnées comme celles des constructions comparatives ont déjà fait l'objet d'études indépendantes. Nous nous limiterons donc à en signaler les points les plus essentiels. Nous suivons Abeillé (2005) en ce qui concerne les coordinations, que nous analysons comme des constructions sans tête avec un sous-constituant [conj $+\mathrm{X}]$ de type tête-complément (cf. figure 1). Nous suivons par ailleurs Desmets (2001) en ce qui concerne la structure interne des ajouts comparatifs non elliptiques, que nous analysons comme des relatives sans antécédent de catégorie SAdv dont la tête, l'adverbe qu-comme, borne la dépendance déclenchée par l'extraction d'un adverbe de manière à l'intérieur de la phrase subséquente, et Desmets (ce numéro) pour ce qui concerne la structure des comparatives elliptiques qui présentent une phrase dont la constituance est fragmentaire, mais dont la sémantique est propositionnelle (cf. figure 2).

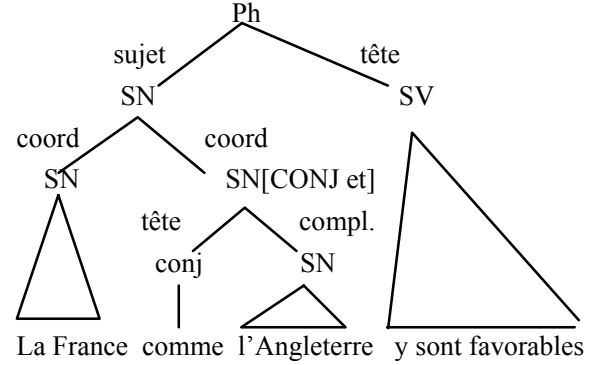

Figure 1

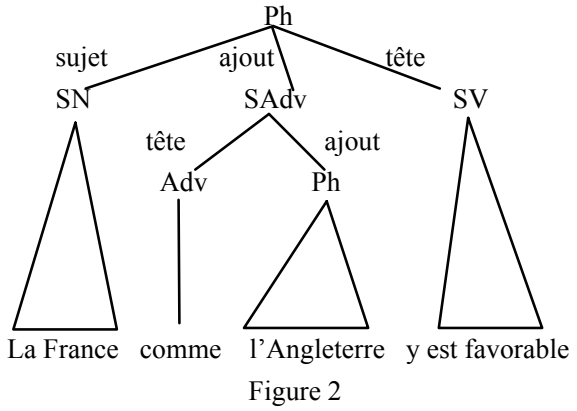

Figure 2 
Il existe une ressemblance de famille générale entre les coordinations de constituants et les structures à ellipse (cf. Mouret 2007), auxquelles appartiennent les comparatives; ce qui explique en partie que les contraintes distributionnelles soient les mêmes dans les deux constructions. D'abord, dans les deux cas, les catégories reliées sont parallèles, et chacune doit respecter les contraintes de sous-catégorisation de l'environnement (cf. 22d), qu'il s'agisse des catégories réalisées de part et d'autre de la conjonction, ou des éléments résiduels de l'ajout comparatif parallèles à des éléments de la phrase matrice :

(22) a. Paul aime [les week-end paisibles à la campagne] / [se balader en ville].

b. Paul aime [les week-end paisibles à la campagne] (,) comme [se balader en ville].

c. Paul apprécie [les week-end paisibles à la campagne] /*[se balader en ville].

d. *Paul apprécie [les week-end paisibles à la campagne] (,) comme [se balader en ville].

Par ailleurs, on observe les mêmes contraintes distributionnelles. Ainsi sont autorisés les phrases, les $\mathrm{SN}$, les SA, les SP, les SAdv et les catégories verbales non conjuguées (cf. 23), mais non les catégories verbales conjuguées (cf. (24) :

(23) a. [Paul dit ce qu'il fait] (,) comme [il fait ce qu'il dit]. (Ph)

b. La mesure concerne [les professions salariées] (,) comme [les professions libérales]. (SN)

c. Il était [fort en français] (,) comme [fort en physique]. (SA)

d. L'hôpital a besoin [de moyens] (,) comme [de personnel]. (SP)

e. Il se croit [légalement] (,) comme [moralement] (,) responsable. (Sadv)

f. Elle adore [recevoir] (,) comme [être invité]. (SV infinitif)

(24) h. *Paul [dit ce qu'il fait] (,) comme [fait ce qu'il dit]. (SV conjugué)

i. *Paul [it] (,) comme [parle] l'anglais couramment. (V conjugué).

On sait que le matériel manquant doit obligatoirement inclure le verbe fini dans une structures à ellipse, ce qui explique immédiatement la restriction observée lorsque la séquence introduite par comme s'analyse comme une comparative. On ne voit pas en revanche ce qui interdit cette combinatoire lorsque la séquence s'analyse comme un conjoint sans ellipse. Nous admettrons ici qu'il s'agit d'une restriction lexicale propre à la conjonction comme, à l'image de ce que l'on observe avec la forme car, (cf. (25a), qui présente bien par ailleurs les propriétés caractéristiques des conjonctions (cf. 25b,c,d) :

(25) a. Paul pleure car*(il) est triste.

b. Paul pleure car il est triste et il est fatigué / *car il est triste et car il est fatigué.

c. Paul pleure car précisément il est triste / *précisément car il est triste.

d. *Car il est triste, Paul pleure. 


\section{Propriétés sémantiques et pragmatiques}

Nous envisageons à présent le fonctionnement interprétatif des deux constructions. Nous rappelons brièvement en \$3.1 les propriétés sémantiques des comparatives en général, puis nous abordons successivement la question de la contribution des comparatives d'analogie en $\$ 3.2$ et des tours intégrés en $\$ 3.3$.

\subsection{Rappels sur l'interprétation des comparatives ${ }^{11}$}

Commune aux divers types de comparatives en comme, on trouve une relation d'identité ou de similarité entre deux propriétés ou 'qualités', respectivement associées à un élément comparé et à un élément comparant qui doivent être de même type sémantique. L'élément comparé est donné par le constituant auquel la construction en comme est adjointe (ou, dans certains cas, auquel elle se combine en tant que complément, cf. Paul s'est comporté comme on peut l'imaginer) et le comparant par la phrase introduite par comme (donné par l'ensemble de la phrase comparative ou par le constituant dont dépend un adverbe de manière extrait).

Amsili et al. (2008), formalisant la sémantique de comme comparatif, en font un opérateur à deux arguments. Comme attendu, le premier argument correspond à la relation sémantique de l'élément avec lequel la comparative se combine. Le second argument est toujours une proposition, de laquelle (ou d'un argument de laquelle) est abstraite une propriété (ce que nous avons appelé ici 'qualité'). L'opération sémantique qu'effectue comme est d'attribuer cette propriété au premier argument ${ }^{12}$.

Selon le type sémantique du constituant auquel la construction en comme est associée, les 'sujets de la comparaison' s'inscrivent dans des dimensions différentes, et l'interprétation de la qualité comparée connaît elle aussi des 'facettes' interprétatives distinctes. On repère ainsi des variations selon qu'une comparative porte sur un verbe, un adjectif, un nom, ou sur une phrase, comme l'illustre le tableau 1. Les comparatives d'analogie que nous avons contrastées jusqu'à présent avec nos tours intégrés relèvent du dernier type.

\footnotetext{
11 Nous reprenons brièvement dans cette sous-section les généralisations de Desmets (2001, chp. 4). Pour une approche différente, voir Moline (2001, 2008b).

${ }^{12}$ Ce calcul diffère légèrement de l'analyse de Desmets (2001) qui propose que comme mette en relation deux propriétés respectives des éléments comparé et comparant. On maintient ainsi l'idée d'une relation de similarité entre les deux propriétés ; alors que pour Amsili et al. (2008) il y a identité. Nous ne tranchons pas ici cette question qui n'a pas d'incidence majeure sur les analyses présentées.
} 


\begin{tabular}{|c|c|c|l|}
\hline Constituant & $\begin{array}{c}\text { Type sémantique } \\
\text { des objets comparés }\end{array}$ & facette de la qualité & \multicolumn{1}{|c|}{ Exemples } \\
\hline Verbe & Événement & «manière » & Il ment comme il respire. \\
\hline Adjectif & Propriété & $\begin{array}{c}\text { Degré, qualité de } \\
\text { réalisation, intensité }\end{array}$ & $\begin{array}{l}\text { Paul est grand comme son } \\
\text { frère est laid. } \\
\text { Paul est intelligent comme } \\
\text { quelqu'un qui a fait de longues } \\
\text { études, mais c'est tout. }\end{array}$ \\
\hline Nom & Individu & Propriété & $\begin{array}{l}\text { des bonbons comme en } \\
\text { achetait ma grand-mère } \\
\text { un anniversaire comme les } \\
\text { organisait mon voisin }\end{array}$ \\
\hline Phrase & Proposition & $\begin{array}{l}\text { Valeur modale } \\
\text { (degré de certitude) }\end{array}$ & $\begin{array}{l}\text { Pierre fait son marché tous les } \\
\text { jeudis, comme Paul tous les } \\
\text { lundis. }\end{array}$ \\
\hline
\end{tabular}

Tableau 1 - Dimensions et facettes de la comparaison en comme

\subsection{Contribution de la comparative d'analogie}

La particularité des comparatives d'analogie est d'associer deux propositions, d'où un effet de parallélisme et de similitude entre les deux événements ou les deux états décrits ${ }^{13}$. La propriété support de la comparaison est modale, plus précisément, elle met en jeu le degré de certitude du locuteur quant à la «satisfiabilité » des propositions; on peut gloser la relation par « $\mathrm{P}$ est vraie de même que/ de la même façon que $\mathrm{Q}$ est vraie » ou bien «il est aussi approprié de dire P est vraie que de dire Q est vraie »; autrement dit, leurs probabilités d'être chacune satisfaite sont jugées égales ou très voisines (où «satisfait » signifie être vérifié dans le monde réel).

Ceci explique que la comparaison d'analogie n'admette pas que les propositions aient deux opérateurs de modalité différents, chaque opérateur imposant des conditions de satisfaction différentes :

(26) a. Personne n'est à la maison. ??Paul est peut-être parti au travail, comme Jean est à l'école.

b. ??Paul est nécessairement parti au travail, comme Jean est probablement parti à l'école.

c. ??Paul est probablement au travail, comme Jean est assurément/ nécessairement à l'école.

\footnotetext{
13 Au delà de la sémantique propre à comme, qui associe deux arguments de même type sémantique, ajoutons que le parallélisme est une caractéristique constructionnelle générale, à l'œuvre tant dans les comparatives (scalaires et non scalaires) que dans les coordinations de propositions (cf. Amsili et al. 2008). Il impose une structure de dépendance du verbe similaire, voire identique, dans chacune des propositions. Cette contrainte explique en particulier la possibilité d'avoir des réalisations fragmentaires dans la seconde phrase, elle en est la condition nécessaire. Elle expliquerait peut-être également le fait que les cas d'analogie comme en (i), où un événement est comparé à un état, soient sémantiquement mal formés :
}

(i) \#Dans cette famille, le père est français, comme la mère a éternué bruyamment. 
Les comparatives d'analogie en comme sont des ajouts parenthétiques ${ }^{14}$ (au sens de Jayez \& Rossari 2004, Bonami \& Godard 2004, Potts 2005) ${ }^{15}$, c'est-à-dire qu'ils n'entrent pas dans les conditions de vérité de la proposition dénotée par la phrase matrice, et inversement, la contribution de la proposition principale est indépendante de celle véhiculée par l'ajout. Typiquement, la construction échappe à la portée des opérateurs sémantiques. Dans les exemples suivants, la phrase matrice est vraie dans les mêmes conditions en (a) et en (b) :

(27) a. Comme sa sœur, Gaston n’est pas silencieux.

b. Gaston n'est pas silencieux.

En (27), l'interprétation de (a) peut être glosée par: il n'est pas vrai que l'individu 'Gaston' a la propriété 'd'être silencieux', de même qu'il n'est pas vrai que l'individu 'sa sœur' a la propriété 'd'être silencieux'. Bien que l'ajout contienne également une négation, il n'a aucune contribution au fait que l'individu 'Gaston' n'a pas la propriété 'd'être silencieux'. De la même façon, en (28a), la proposition d'analogie n'appartient pas au contenu questionné ; seule, la proposition principale est sous l'opérateur. Pour que la comparative soit également questionnée, il faudrait utiliser un opérateur qui porte sur l'énoncé lui-même, soit : est-il vrai que (de même que/ comme) son oncle est déprimant, Jean est déprimant?

(28) a. Comme son oncle, Jean est-il un garçon déprimant?

b. Jean est-il un garçon déprimant?

Le contenu de l'ajout d'analogie appartient à l'arrière plan, il n'est pas intégré au niveau de l'assertion principale, c'est-à-dire au niveau de ce qui est en discussion (ce que Potts 2005 appelle le contenu «at-issue »). Sa contribution, a lieu au niveau des relations de discours, autrement dit, au niveau de l'énoncé même. Plusieurs propriétés empiriques, typiques des éléments assertés en arrière plan, témoignent du fait que son contenu n'est pas directement accessible ${ }^{16}$. On signale ici l'impossibilité de remettre en cause le contenu de l'ajout incident dans le dialogue :

\footnotetext{
14 Il existe une autre forme parenthétique de comparative en comme, appelée ajout reportif, utilisée pour mettre en mention ou rapporter un énoncé (La bourse s'est effondrée, comme l'avait prédit Le Monde; des discours "politiquement corrects », comme disesnt les Américains). Sémantiquement, la relation permet de mettre en conformité deux actes de langages (celui de l'énoncé rapporté et celui qui le rapporte) Les propriétés de l'ajout reportif sont semblables à celles de l'ajout d'analogie : il n'y pas de contribution au niveau de la proposition dénotée par la phrase matrice (le contenu de l'ajout n'entre pas dans ses conditions de vérité), mais la contribution se fait au niveau de l'énoncé et des relations de discours. Pour plus de détails voir Roussarie \& Desmets, 2003.

${ }^{15}$ Si la comparative d'analogie est parenthétique, toutefois, elle n'est pas «speaker oriented», à la différence des adverbes d'énoncés (cf. Bonami \& Godard 2006, Potts 2005).

${ }^{16}$ Nous citons ci-après la remarque qu'un relecteur nous a faite (qu'il en soit ici remercié) à propos des effets d'ironie découlant de l'utilisation d'une proposition comparante fausse, et qui confirme notre analyse sémantique de la comparative d'analogie :
}

(i) Tu parles, ce type est mexicain, comme moi je suis russe! 
(29) A : la France, comme d'ailleurs l'Angleterre, a voté ce projet.

B : \# Non, l'Angleterre n'a pas voté ce projet.

C : Parce que l'Angleterre était impliquée dans le projet ? Je ne le savais pas.

Pour accéder au contenu de la proposition véhiculée par la comparative, il est nécessaire de procéder à une rupture discursive (Jayez 2004), ce que réalise (29c).

De même, il est assez difficile d'inclure l'ajout incident dans l'interprétation d'une reprise anaphorique de la phrase matrice. En (30), l'interprétation la plus saillante est que le pronom anaphorique le n'inclut pas l'ajout en comme.

(30) Comme son oncle, Paul est un garçon déprimant; il l'est depuis l'enfance.

\subsection{Contribution des tours intégrés}

La différence entre la sémantique du tour intégré en comme et celle de l'ajout incident est liée à leur mode de contribution. Alors que le contenu de l'incident est en arrière plan, qu'il n'entre pas dans les conditions de vérité de la proposition véhiculée par la phrase matrice, et que sa contribution sémantique se réalise au niveau de l'énoncé, le tour intégré, lui, est une des conditions à vérifier pour que la proposition dans son ensemble soit vraie. Autrement dit, sa contribution est incluse dans l'assertion principale.

\subsubsection{De l'analogie dans la coordination?}

Nous faisons l'hypothèse que comme-coordonnant conserve certains traits sémantiques de comme-adverbe comparatif: il discrimine deux éléments (ses arguments sémantiques) qu'il met sur un plan d'égalité, d'où l'effet d'analogie que l'on perçoit aussi avec commecoordonnant. En plus d'être conforme à l'intuition, ceci permet d'expliquer quelques particularités combinatoires du tour intégré.

Premièrement, à l'image de comme-comparatif, comme-coordonnant est un opérateur strictement binaire. Contrairement aux coordinations additives ordinaires qui ne limitent pas le nombre de conjoints qu'elles associent, comme ne coordonne que deux éléments.

(31) a. *La France, l'Espagne comme l'Angleterre sont dans la ligne de mire.

b. La France, l'Espagne et l'Angleterre sont dans la ligne de mire.

En (i), il y a un décalage entre le contenu de la comparative, qui n'est pas asserté, et le contenu de la principale, qui lui l'est. En « disant « $p$, comme q » le locuteur affirme que $p$ est vrai et suggère que $p$ et $\mathrm{q}$ ont les mêmes conditions de satisfiabilité-ce faisant il suggère donc que q est vrai. Et c'est précisément cela qui provoque l'effet d'ironie en (i). Si (i) était un acte illocutoire sincère [i.e. sans ironie], il serait problématique que le locuteur affirme d'une part que la proposition «ce type est mexicain » est vraie, et suggère d'autre part que cette proposition a le même degré de satisfiabilité que « je suis russe». Or, dans le contexte, il est clair que le locuteur n'est pas russe, il ne peut donc pas être sincère quand il affirme «ce type est mexicain ». » 
Deuxièmement, les arguments de comme-coordonnant sont obligatoirement discriminés ${ }^{17}$, de sorte qu'aucune interprétation de groupe n'est autorisée, à la différence de ce que l'on observe dans divers contextes avec la conjonction et (cf. Link 1983, Landman 1989).

En (32a), Paul et Marie désignent deux individus ou alternativement une entité couple, ce qui autorise l'ambiguité sur le nombre de voitures achetée(s). Or, une telle ambiguité n'est pas possible dans la coordination en comme (32b).

(32) a. Paul et Marie ont acheté une voiture. (une voiture ou deux voitures achetées)

b. ?Paul comme Marie ont acheté une voiture. (deux voitures achetées)

En (33a), seule la lecture de groupe est autorisée, laquelle n'est pas possible en (33b) avec comme coordonnant :

(33) a. Paul et Marie forment un beau couple.

b. *Paul comme Marie forment un beau couple

De même, en (34), où deux N/N' coordonnées partagent un seul déterminant, seule l'interprétation de groupe est possible (on désigne là l'ensemble des individus qui possèdent la propriété d'être N1 et la propriété d'être N2) et à nouveau, comme coordonnant est exclu.

(34) a. En cas de guerre, les médecins et réservistes seront réquisitionnés en priorité.

b. *En cas de guerre, les médecins comme réservistes seront réquisitionnés en priorité.

On observe enfin le même genre d'impossibilité lorsqu'un SN complément de Prép est formé de deux pronoms forts ou de deux noms propres coordonnés :

(35) a. La honte tombera sur vous et moi / sur Paul et Marie.

b. *La honte tombera sur vous comme moi / sur Paul comme Marie.

Troisièmement, contrairement à d'autres coordinations additives, les éléments de part et d'autre de comme ne peuvent pas entretenir de relation de discours asymétriques de type succession narrative (cf. 36), cause-conséquence, etc. (cf. Kehler 2002, Mouret 2007). Lexicalement, comme-coordonnant est doté d'une relation qui met sur un plan d'égalité les éléments distingués : il y a parallélisme et non succession, équivalence des événements décrits et non interdépendance (de type cause-conséquence).

(36) a. Il recevra les étudiants de première année comme (*ensuite) les étudiants de deuxième année

b. Il recevra les étudiants de première année et (ensuite) les étudiants de deuxième année

\footnotetext{
17 On fait l'hypothèse que cette propriété provient de la sémantique du comme comparatif, puisque discriminer deux éléments est une condition inhérente à la sémantique de la comparaison.
} 
Au vu de ces propriétés combinatoires, on peut être tenté d'élargir l'hypothèse en disant que comme-coordonnant et comme-comparatif sont en réalité porteurs de la même relation sémantique. La seule différence concernant la sémantique des tours intégrés proviendrait du fait que la coordination en comme manipule deux fonctions propositionnelles (permettant de construire une proposition à partir du contenu de chaque conjoint) ${ }^{18}$ comme arguments, plutôt que deux propositions. Une fois les propositions reconstruites, la relation de similarité propre à comme, qui passe par l'abstraction d'une propriété (du second argument) attribuée au premier argument s'applique naturellement. De fait, si l'on peut gloser rapidement la sémantique d'une coordination de propositions par «P est vraie et $\mathrm{Q}$ est vraie aussi », on voit aisément que les conditions de statisfiabilité identiques/semblables de $\mathrm{P}$ et $\mathrm{Q}$, qui déterminent l'interprétation d'analogie, sont réunies.

\subsubsection{Une contribution intégrée}

Alors que le contenu de l'ajout incident appartient à l'arrière plan, le contenu du conjoint introduit par comme se trouve asserté au même niveau que les autres éléments de la proposition. Il entre dans les conditions de vérité de la proposition et est accessible à diverses opérations. Par exemple, il est possible de remettre en cause le contenu du conjoint en comme dans le dialogue :

(37) A : la France comme l'Angleterre ont voté ce projet.

B : C'est faux ! L'Angleterre a refusé au dernier moment.

De plus, la construction est dans la portée des opérateurs de négation ou d'interrogation ${ }^{19}$ :

(38) a. Vous comme moi ne valons pas les grands seigneurs.

b. Paul comme Marie auront-ils rattrapé leur retard à la fin de l'année?

L'interprétation de (38a) peut être glosée par : il n'est pas vrai que les individus 'vous' et 'moi' aient la propriété 'de valoir les grands seigneurs' ; la proposition ne tient que si ni 'vous' ni 'moi' n'ont la propriété en question. En (38b), l'opérateur d'interrogation porte sur deux propositions parallèles, la question peut se gloser par chacun aura-t-il rattrapé son retard à la fin de l'année?

Enfin, il n'y a pas de difficulté à inclure le contenu du conjoint en comme dans l'interprétation d'une reprise anaphorique de la phrase matrice :

(39) La France comme l'Angleterre sont dans la ligne de mire des Etats-Unis; ils l'ont été depuis le début des négociations en n'acceptant pas de ratifier le traité.

Ainsi, la différence entre comme-coordonnant et comme-comparatif ne semble pas se situer au niveau sémantique : le calcul des arguments change légèrement, mais on

\footnotetext{
18 Ce type de résolution est l'analyse «standard » proposée pour la sémantique des coordinations (cf. Gazdar 1980, Partee \& Rooth 1983).

19 On notera toutefois que la négation et l'interrogation sont parfois délicates à obtenir dans ces phrases.
} 
peut faire l'hypothèse que la relation sémantique de comme comparatif demeure la même. $\mathrm{Au}$ niveau pragmatique, en revanche, les tours se distinguent puisque le contenu du conjoint en comme est asserté au même niveau que les autres constituants de la phrase matrice, alors que l'ajout comparatif est présenté comme un commentaire d'arrièreplan.

\section{Comparaison avec les autres formes similatives}

A première vue, le double fonctionnement syntaxique que nous avons mis en évidence est également observé par les formes similatives ainsi que, et plus marginalement de même que (cf. 40). L'accord au pluriel est certes plus naturel avec la première forme (et du reste bien attesté, cf. (41)), mais il ne semble pas cependant exclu avec la seconde (avec des variations dans les acceptabilités). Nous montrons brièvement que les tours intégrés en ainsi que / de même que se comportent comme ceux en comme. Les tours incidents doivent, en revanche, recevoir une autre analyse.

(40) a. La France, ainsi que l'Angleterre est favorable à ce projet.

b. La France ainsi que l'Angleterre sont favorables à ce projet.

c. La France, de même que l'Angleterre, est favorable à ce projet.

d. La France de même que l'Angleterre sont favorables à ce projet.

(41) a. Cette annonce ainsi que la promesse d'organiser un référendum le 28 janvier prochain sur le rétablissement éventuel de la peine de mort sont intervenues à la suite d'une journée de folie qui aurait dû en principe être consacrée à rendre hommage à la mémoire des victimes de la révolution roumaine. (LM)

b. Pour mettre en relief le caractère communautaire de l'entreprise, la Commission européenne ainsi que la Banque européenne d'investissement LRB BEIRB ont été invitées en tant que telles à la réunion de Paris. (LM)

c. Le premier producteur mondial, Inco, ainsi que Falconbridge ont d'ores et déjà procédé à des réductions de production pour s'adapter à la nouvelle physionomie du marché. (LM)

d. «La pérennité de chacun des sites industriels ainsi que leur vocation respective sont confirmées » conclut le texte du gouvernement. (LM)

\subsection{Propriétés des tours intégrés}

On vérifie aisément que les tours intégrés en ainsi que / de même que fonctionnent comme ceux en comme. D'abord, la forme similative se comporte comme une conjonction de coordination : elle ne peut pas être combinée à un autre coordonnant (42a), peut être suivie mais non précédée d'un adverbe connecteur tel que en effet (42b) et ne peut être antéposée à l'initiale de la phrase avec le syntagme qu'elle introduit (42c).

(42) a. La France ainsi que / de même que l'Angleterre et (*ainsi que / *de même que) l'Espagne sont favorables à ce projet. 
b. La France (*en effet) ainsi que / de même que (en effet) l'Angleterre sont favorables à ce projet.

c. *Ainsi que / de même que l'Angleterre, la France sont favorables à ce projet.

Par ailleurs, la construction présente les mêmes propriétés syntaxiques que nos tours intégrés en comme. Sur le plan combinatoire d'abord, les possibilités et les restrictions sont les mêmes, comme l'illustrent les exemples suivants qui reprennent en tous points ceux examinés supra:

(43) a. Paul aime [les week-end à la campagne] / [se balader en ville].

b. Paul aime [les week-end à la campagne] ainsi que / de même que [se balader en ville].

c. Paul aime [les week-end à la campagne] / [se balader en ville].

d. *Paul apprécie [les week-end à la campagne] ainsi que / de même que [se balader en ville].

(44) a. [Paul dit ce qu'il fait] ainsi qu' / de même qu' [il fait ce qu'il dit]. (Ph)

b. La mesure concerne [les professions salariées] ainsi que / de même que [les professions libérales]. (SN)

c. Il est [fort en français] ainsi que / de même que [fort en physique]. (SA)

e. L'hôpital manque [de moyens] ainsi que / de même que [de personnel]. (SP)

d. Elle adore [recevoir] ainsi qu' / de même qu' [être invitée]. (SV infinitif)

f. Il se croit [légalement] ainsi que / de même que [moralement] responsable. (Sadv)

(45) a. *Paul [dit ce qu'il fait] ainsi que [fait ce qu'il dit]. (SV fini)

b. ??Paul [lit] ainsi que [parle] l'anglais couramment. (V fini).

(46) "Grève nationale : professeurs ainsi qu'élèves sont invités à se joindre à la manifestation générale à Paris réunissant fonctionnaires de l'éducation nationale et syndicats." (Web)

Les contraintes concernant l'extraction sont par ailleurs bien observées : seule l'extraction parallèle hors de chaque terme est possible, comme l'illustrent les contrastes suivants :

(47) a. Reste donc une entreprise dont [le président -] ainsi que / de même que [le vice-président -] se plaisent à vanter le chiffre d'affaire.

b. *Reste donc une entreprise dont [le président -] ainsi que / de même que [son vice-président] se plaisent se à vanter le chiffre d'affaire.

c. *Reste donc une entreprise dont [son président] ainsi que / de même que [le vice-président -] se plaisent à vanter le chiffre d'affaire. 
Enfin, on observe les mêmes contraintes sur le plan sémantico-pragmatique : la construction est nécessairement binaire (48a), ne peut donner lieu à une interprétation de groupe (48b) ni mettre en jeu une relation de discours telle que la succession (48c); elle appartient bien par ailleurs au contenu asserté, comme l'illustre (49) :

(48) a. *La France, l'Espagne ainsi que / de même que l'Angleterre sont favorables à ce projet.

b. *La France ainsi que / de même que l'Angleterre forment une équipe.

c. *La France ainsi que / de même qu'ensuite l'Angleterre se sont associées à ce projet.

(49) A : La France ainsi que / de même que l'Angleterre sont favorables à ce projet.

B : C'est faux ! Seule l'Angleterre y est favorable.

Nous en concluons que les tours intégrés en ainsi que / de même que ne se distinguent pas fondamentalement de ceux étudiés dans cet article. Syntaxiquement, la structure s'analyse comme une coordination et la forme similative comme un coordonnant. Sémantiquement, on peut penser que la même relation d'analogie est mise en jeu. Il est possible cependant que les deux tours se distinguent sur le plan informationnel (cf. Huddleton \& Pullum 2002 : 1317 pour une analyse en ce sens de la forme as well as). Nous laisserons ici la question ouverte.

\subsection{Propriétés des tours incidents}

L'analyse des tours incidents en ainsi que / de même que est plus délicate. D'un côté, les contraintes lexicales sont les mêmes que dans les tours intégrés (50), ce qui apparente à nouveau la forme similative à une conjonction. En particulier, l'antéposition du syntagme introduit par ainsi que / de même que à l'initiale de la phrase reste exclue pour la plupart des locuteurs lorsque la séquence est incidente et que le verbe est au singulier (voir cependant Piot 1993, 1995 pour un jugement différent), ce qui constitue un trait typique des coordonnants.

(50) a. La France, ainsi que / de même que l'Angleterre et (*ainsi que / *de même que) l'Espagne, est favorable à ce projet.

b. La France, (*précisément) ainsi que / de même que (précisément) l'Angleterre, est favorable à ce projet.

c. *Ainsi que / de même que l'Angleterre, la France est favorable à ce projet.

D’un autre côté, la construction dans son ensemble ne se comporte pas comme une coordination : les données de l'extraction sont les mêmes que dans les structures tête-ajout (51), de même que les données combinatoires (52-54), les SN sans déterminant étant exclus (55).

(51) a. Reste donc une entreprise dont [le président -], ainsi que / de même que [le vice-président -], se plaît à vanter le chiffre d'affaire. 
b. Reste donc une entreprise dont [le président -], ainsi que / de même que [son vice-président], se plait se à vanter le chiffre d'affaire.

c. *Reste donc une entreprise dont [son président], ainsi que / de même que [le vice-président -], se plait à vanter le chiffre d'affaire.

(52) a. [Paul dit ce qu'il fait], ainsi que / de même que d'ailleurs [il fait ce qu'il dit]. (Ph)

b. La mesure concerne [les professions salariées], ainsi que / de même que d'ailleurs [les professions libérales]. (SN)

c. Il est [fort en français], ainsi que / de même que d'ailleurs [fort en physique]. (SA)

e. L'hôpital a besoin [de moyens], ainsi que / de même que d'ailleurs [de personnel]. (SP)

d. Elle adore [recevoir], ainsi qu' / de même que d'ailleurs [être invité e]. (SV infinitif)

f. Il se croit [légalement], ainsi que / de même que d'ailleurs [moralement], responsable. (Sadv)

(53) a. *Paul [dit ce qu'il fait], ainsi que d'ailleurs [fait ce qu'il dit]. (SV fini)

b. ??Paul [lit], ainsi que d'ailleurs [parle] l'anglais couramment. (V fini).

(54) a. Paul aime [les week-end à la campagne] / [se balader en ville].

b. Paul aime [les week-end à la campagne], ainsi que / de même que d'ailleurs [se balader].

c. Paul apprécie [les week-end à la campagne] / *[se balader en ville].

d. *Paul apprécie [les week-end à la campagne], ainsi que / de même que d'ailleurs [se balader en ville].

(55) *Grève nationale : professeurs, ainsi qu'élèves d'ailleurs, sont invités à se joindre à la manifestation générale à Paris réunissant fonctionnaires de l'éducation nationale et syndicats.

Une solution a été proposée pour rendre compte de ce paradoxe apparent (cf. Abeillé 2005) : elle consiste à admettre qu'un syntagme introduit par une conjonction n'est pas nécessairement coordonné (56a); il peut alternativement être ajout incident à la phrase (56b-f).

(56) a. La France et l'Angleterre sont favorables à ce projet.

b. La France est favorable à ce projet, et l'Angleterre aussi.

c. La France est favorable, et l'Angleterre aussi, à ce projet.

d. La France est, et l'Angleterre aussi, favorable à ce projet.

e. La France, et l'Angleterre aussi, est favorable à ce projet.

f. *Et l'Angleterre aussi, la France est favorable à ce projet. 
François Mouret \& Marianne Desmets

C'est l'analyse que nous admettrons ici: ainsi que et de même que sont ainsi recatégorisés comme des conjonctions dans tous leurs emplois non phrastiques, mais ils peuvent, à la différence de comme, fonctionner aussi bien comme membre coordonné que comme ajout incident.

\section{Conclusion}

Nous avons montré que les cas d'accord sujet-verbe de certaines suites en SN comme $\mathrm{SN}$ ne sont pas un simple effet du parallélisme sémantique entre deux propositions mais correspondent à l'existence d'une construction coordonnée en comme, où l'adverbe connaît une recatégorisation en conjonction. Si la sémantique de cette coordination et celle la comparative d'analogie présentent des similarités, en revanche, il existe un contraste pragmatique et prosodique net entre les deux constructions. Ce double fonctionnement est propre à comme et ne se retrouve pas tel quel avec les locutions similatives ainsi que / de même que qui fonctionnent comme des coordonnants dans tous leurs emplois non phrastiques.

\section{RÉFÉRENCES BIBLIOGRAPHIQUES}

ABEILlÉ, A. (2005), «Les syntagmes conjoints et leurs fonctions syntaxiques », Langages 160, pp. $42-66$.

ABeillé, A., Clément, L. \& F. Toussenel (2003), Building a French treebank. In A. Abeillé (ed), Treebanks. Dordrecht : Kluwer. 165-188.

Amsili, P. \& M. Desmets (2008), «French Comparative Ellipsis», International Conference on Elliptical constructions, LLF \& Université Paris Diderot, Chicago Center, Paris.

AsHeR, N. (2000), «Truth conditional discourse semantics for parentheticals », Journal of Semantics 17(1), pp. 31-50.

BÈGue, D. (1977), Quelques aspects de la coordination en français. Thèse de Doctorat, Université Paris 7.

Blanche-Benveniste, C. \& A. Chervel (1966), « Recherches sur le syntagme substantif», Cahiers de Lexicologie 9, pp. 3-37.

BonAmi, O., Godard, D. \& B. Kampers-Manhe (2004), «Adverb classification », in F. Corblin et H. de Swart (éds), Handbook of French semantics. Stanford : CSLI Publications, pp. 143-183.

BRESNAN, J. \& J. Grimshaw (1978), «The syntax of free relatives in English », Linguistic Inquiry 9-3, pp. 331-391.

CuRAT, H. (1999), Les déterminants dans la référence nominale et les conditions de leur absence, Genève/ Paris, Droz.

Desmets, M. (2001), Les typages de la phrase en HPSG : le cas des phrases en comme. Thèse de doctorat, Université Paris X- Nanterre. 
Desmets, M. (à paraitre), «Les phrases à extraction», in A. Abeillé, A. Delaveau \& D. Godard (éds.), Grande Grammaire du Français, chp1. (coord.) A. Delaveau. Bayard Presse.

AmsiLi, P., Desmets, M., Roussarie, L. \& J. Tseng (à paraitre), « Comparatives et consécutives », chp15. (coord.) M. Desmets, in Delaveau, A. et Godard, D. (éds.) Grande Grammaire du Français. Bayard Presse.

GoldSMith, J. (1985), «A principled exception to the coordinate structure constraint ». Chicago Linguistic Society 20, pp. 133-143.

GAZDAR, G. (1980), «A cross-categorial semantics for conjunction». Linguistics and Philosophy 3, pp. 407-409.

Grevisse, M. (1936), Le bon usage. Douzième édition refondue par A. Goosse (1986), Paris, Duculot.

Huddelston, R. \& G. Pullum (2002), The Cambridge Grammar of the English Language. Cambridge : Cambridge University Press.

JAYEZ, J. (2004), Notes de cours sur la présupposition. ms. ENS Lyon.

JAYEZ, J. \& C. Rossari (2004), «Parentheticals as conventional implicatures », in F. Corblin \& H. de Swart (éds), Handbook of French semantics. Stanford : CSLI Publications, pp. 211-231.

KeHLer, A. (2002), Coherence, reference and the theory of grammar. Stanford: CSLI Publications.

LAKOFF, G. (1986), «Frame semantic control of the coordinate structure constraint ». Chicago Linguistic Society 22, pp. 152-167.

Landman, F. (1989), Groups. Language and Philosophy 12.5 \& Language and Philosophy 12.6, pp. 599-605 \& 723-744.

LE GOFFIC, P. (1991), « Comme, adverbe connecteur intégratif : éléments pour une description », Travaux linguistiques du CERLICO 4, pp. 11-31. Rennes, Presses Universitaires de Rennes 2.

LINK, G. (1983), The logical analysis of plurals and mass terms : a lattice-theoretic approach. In R. Bauerle, C. Schwarze \& A. von Stechow (eds), Meaning, use and interpretation of language. Berlin : Mouton de Gruyter, pp. 302-323.

Moline, E. (2001), «Elle ne fait rien comme tout le monde. Les modifieurs adverbiaux de manière en comme », Revue Romane 36-2, Copenhague, pp 171-192.

MoLINE, E. (2008), «Elle volait pour voler, comme on aime pour aimer : les propositions d'analogie en comme », in E. Moline \& N. Flaux (éds), Langue Française 159, 83-99.

MOURET, F. (2007), Grammaire des constructions coordonnées. Coordinations simples et coordinations à redoublement en français contemporain. Thèse de doctorat, Université Paris 7.

PARTEE, B. \& M. Rooth (1983), «Generalized conjunction and type ambiguity », R. Bauerle, C. Schwarze, et A. von Stechow (éds.), Meaning, use, and interpretation of Language. Berlin : Walter de Gruyter, pp. 361-383.

PiOT, M. (1993), «Les connecteurs du français », Lingvisticae Investigationes 17-1, pp. 141-160.

РіОт, М. (1995), Composition transformationnelle de phrases par subordination et coordination. Thèse d'État, Université Paris 7.

POTTS, C. (2005), The Logic of Conventional Implicatures. Oxford University Press. 
François Mouret \& Marianne Desmets

RoOdenburg, J. (2005), «Une coordination particulière : les syntagmes $N^{\prime}$ Conj $N^{\prime}$ en français », Langages 160, pp. 93-109.

Roussarie, L. \& M. Desmets, M. (2003), "Quotative reference in reportive comme clauses », in Beyssade C., Bonami O., Cabredo Hofherr P. \& Corblin F. (éds), Empirical Issues in Formal Syntax and Semantics 4 Paris : Presses Universitaires de Paris-Sorbonne, pp. 329-344.

Ross, J. R. (1967), Constraints on variable in syntax, Ph.D., MIT. 